\title{
SOME RESULTS ON DIFFERENTIABLE ACTIONS
}

\author{
BY WU-CHUNG HSIANG AND WU-YI HSIANG ${ }^{1}$
}

Communicated by W. S. Massey, September 7, 1965

In this note, we shall announce some results on differentiable actions of $\mathrm{SO}(n), \mathrm{SU}(n)$ and $\mathrm{Sp}(n)$ on manifolds. Since the detailed proofs are too long to be included here, we shall publish them elsewhere.

THEOREM 1. Let $\phi$ be a differentiable action of $\mathrm{SO}(n),(\mathrm{SU}(n)$, $\mathrm{Sp}(n))$ on an $m$-dim manifold $M^{m}$ where $n \geqq 11$ and $m \leqq(n-1)^{2} / 4$ $\left(n \geqq 8\right.$ and $m \leqq(n-1)^{2} / 2, n \geqq 8$ and $\left.m \leqq(n-1)^{2}\right)$. If the first rational Pontrjagin class of $M^{m}, P_{1}\left(M^{m}\right)$, vanishes, then the identity component of any isotropy subgroup, $\left(G_{x}\right)_{0}$ for $x \in M^{m}$ is always conjugate to $\mathrm{SO}(k)$, $(\mathrm{SU}(k), \mathrm{Sp}(k))$ under the standard inclusion for some $k \geqq \frac{2}{3} n$.

TheOREm 2. For a given differentiable action $\phi$ of $\mathrm{SO}(n),(\mathrm{SU}(n)$, $\mathrm{Sp}(n)$ ) on a homotopy sphere $\Sigma^{m}$ (respectively Euclidean space $R^{m}$, respectively disc $\left.D^{m}\right)$ where $n \geqq 11$ and $m \leqq(n-1)^{2} / 4 \quad(n \geqq 8$ and $m \leqq(n-1)^{2} / 2, n \geqq 8$ and $\left.m \leqq(n-1)^{2}\right)$, we have that

(i) all orbits are real (complex, quaternionic) Stiefel manifolds,

(ii) if $\mathrm{SO}(n) / \mathrm{SO}(k),(\mathrm{SU}(n) / \mathrm{SU}(k), \mathrm{Sp}(n) / \mathrm{Sp}(k))$ is the principal orbit and $F$ is the fixed point set, then

$$
\begin{aligned}
H^{*}(F ; A) & \simeq H^{*}\left(S^{\gamma} ; A\right) \\
\left(\text { respectively } H^{*}(F ; A)\right. & \simeq H^{*}\left(R^{\gamma} ; A\right) \\
\text { respectively } H^{*}(F ; A) & \left.\simeq H^{*}\left(D^{\gamma} ; A\right)\right)
\end{aligned}
$$

where

$$
\begin{aligned}
\gamma & =\operatorname{dim} F=m-n(n-k) \text { for the } \mathrm{SO}(n) \text { case } \\
& =m-2 n(n-k) \text { for the } \mathrm{SU}(n) \text { case } \\
& =m-4 n(n-k) \text { for the } \mathrm{Sp}(n) \text { case }
\end{aligned}
$$

and

$$
\begin{aligned}
A & =Z_{2} \quad \text { for the } \mathrm{SO}(n) \text { case }(n \text { odd }) \\
& =Z \quad \text { for the other cases. }
\end{aligned}
$$

\footnotetext{
1 During the preparation of this paper, the first named author was partially supported by NSF grant NSF-GP-4037 and the second named author was partially supported by NSF grant NSF-GP-3422.
} 
(iii) the orbit space $\Sigma^{m} / \phi$ is a $(k-1)$-connected ((2k-1)-connected, $(4 k-1)$-connected) differentiable manifold with boundary and the boundary is the image of singular orbits.

Let $\left(H_{1}\right),\left(H_{2}\right)$ be two conjugate classes of connected subgroups of $G$ such that $H_{2} / H_{1} \simeq S^{k}(k \neq 1,3)$. We shall give a complete set of invariants for differentiable actions of $G$ on compact connected manifolds with $\left(H_{1}\right),\left(H_{2}\right)$ as the isotropy subgroup types. Recall that $H_{2} / H_{1} \simeq S^{k}(k \neq 1,3)$. In fact, it follows from the Slice Theorem [1], [11] that $H_{2} / H_{1}$ must be diffeomorphic to $S^{k}$ for some $k$ if there exists an action of $G$ with $\left(H_{1}\right),\left(H_{2}\right)$ as the isotropy subgroups.

A pre- $G$-space with $\left(H_{1}\right),\left(H_{2}\right)$ as the isotropy subgroup types is an object

$$
\Phi=\{B, \xi, \zeta,(f: \hat{\eta} \rightarrow \hat{\xi} \mid L),(g: \hat{\eta} \rightarrow \xi)\}
$$

satisfying the following four conditions:

(i) $B$ is an $n$-dim compact connected manifold with boundary and $\xi$ is a differentiable $G / H_{1}$-bundle over $B$ with $N\left(H_{1}\right) / H_{1}$ as the structural group. $\hat{\xi}$ is the principal $N\left(H_{1}\right) / H_{1}$-bundle associated with $\xi$.

(ii) $\zeta$ is a differentiable $G / H_{2}$-bundle over a $(n-1)$-dim regular compact submanifold $L$ of $\partial B$ ( $L$ may be disconnected) with $N\left(H_{2}\right) / H_{2}$ as the structural group. $\hat{\zeta}$ is the principal $N\left(H_{2}\right) / H_{2}$-bundle associated with $\zeta$.

(iii) The structural group of $\xi \mid L$ is reduced to $\left(N\left(H_{1}\right) \cap N\left(H_{2}\right)\right) / H_{1}$ and $f: \hat{\eta} \rightarrow \hat{\xi}$ is the reduction.

(iv) There is a bundle map $g: \hat{\eta} \rightarrow \xi$ which induces the natural homomorphism $\left(N\left(H_{1}\right) \cap N\left(H_{2}\right)\right) / H_{1} \rightarrow N\left(H_{2}\right) / H_{2}$ on the fibre and identity on the base $L$.

Notice that for a given principal $\left(N\left(H_{1}\right) \cap N\left(H_{2}\right)\right) / H_{1}$-bundle $\hat{\eta}$, $g$ is unique if it exists. We define isomorphism classes of pre- $G$-spaces in the natural way and only consider isomorphism classes of pre-Gspaces.

TheOREM 3. To each differentiable action $\phi$ of $G$ on a compact connected manifold $M$ with $\left(H_{1}\right),\left(H_{2}\right)$ as isotropy subgroups, there corresponds a unique isomorphism class of pre-G-space

$$
\Phi=\{B, \xi, \zeta,(f: \hat{\eta} \rightarrow \xi \mid L),(g: \hat{\eta} \rightarrow \xi)\}
$$

such that

(i) the orbit space $M / \phi$ is diffeomorphic to $B$,

(ii) $\xi \mid(B-L)$ is the differentiable fibre bundle

$$
\frac{G}{H_{1}} \rightarrow M_{\left(H_{1}\right)} \rightarrow(B-L)
$$


where $M_{\left(H_{1}\right)}$ is the union of all principal orbits,

(iii) $\zeta$ is the differentiable fibre bundle

$$
\frac{G}{H_{2}} \rightarrow M_{\left(H_{2}\right)} \rightarrow L
$$

where $M_{\left(H_{2}\right)}$ is the union of all singular orbits.

Conversely, for a given pre-G-space $\Phi$, there is a unique equivalence class $\phi$ of differentiable action of $G$ on a compact connected manifold $M$ such that the above conditions (i), (ii) and (iii) are verified.

Consider the set of all equivalence classes of differentiable actions of $G$ on compact connected manifolds with $\left(H_{1}\right),\left(H_{2}\right)$ as the isotropy subgroup types such that the associated pre- $G$-spaces have isomorphic $\xi, \zeta$ and diffeomorphic $B, L$. Since $g$ is completely determined by $f$ as we remarked before, the actions are completely distinguished by the reductions $f: \hat{\eta} \rightarrow \hat{\xi} \mid L$.

Under these circumstances, the reduction $f: \hat{\eta} \rightarrow \hat{\xi} \mid L$ is called the "twist invariant" of a differentiable $G$-action $\phi$. By [14], the "twist invariant" is represented as a homotopy class of cross-sections of the fibre bundle

$$
N\left(H_{1}\right) /\left(N\left(H_{1}\right) \cap N\left(H_{2}\right)\right) \rightarrow E(\xi \mid L) /\left(N\left(H_{1}\right) \cap N\left(H_{2}\right)\right) \rightarrow L .
$$

Applying Theorem 3 to fixed point free actions of $\mathrm{SO}(n)(\mathrm{SU}(n)$, $\mathrm{Sp}(n))$ on $(2 n-1)-((4 n-1)-,(8 n-1)-1)$ spheres, we generalize the classification theorem of [5].

THEOREM 4. Let $\phi$ be a fixed point free differentiable action of $\mathrm{SO}(n)$, (SU $(n), \mathrm{Sp}(n))$ on a homotopy sphere $\Sigma^{2 n-1}\left(\Sigma^{4 n-1}, \Sigma^{8 n-1}\right)$ for $n \geqq 11$ $(n \geqq 8)$. Then the orbit space is always a $D^{2}$ (3-dim compact contractible manifold, 5-dim compact contractible manifold). Moreover, the $\mathrm{SU}(n)$ and $\mathrm{Sp}(n)$ actions are completely determined by the orbit spaces while all such $\mathrm{SO}(n)$ actions are distinguished by their "twist invariants" which may be identified as elements in $H^{1}\left(S^{1} ; Z\right)$. For the latter case, the "twist invariant" is always a generator of $H^{1}\left(S^{1} ; Z\right)$ if $n$ is even and it can be any odd multiple of a generator of $H^{1}\left(S^{1} ; Z\right)$ if $n$ is odd.

Now, let us study differentiable actions of compact connected classical groups on spheres with three types of orbits such that one of them is fixed point.

Let $\phi$ be a differentiable action of $\mathrm{SO}(n)(\mathrm{SU}(n), \mathrm{Sp}(n))$ on a homotopy sphere $\Sigma^{m}$ for $m<3 n-6(m<6 n-9, m<12 n-15)$. By Theorem 2 and Theorem 4 , the only remaining case is that where $2 n-1<m<3 n-6 \quad(4 n-1<m<6 n-9,8 n-1<m<12 n-15)$ and 
$V_{n, 2}\left(W_{n, 2}, X_{n, 2}\right)$ is the principal orbit type. In this case, the fixed point set is nonempty and of dimension $m-2 n(m-4 n, m-8 n)$. It again follows from Theorem 2 that the orbit space of $\phi$ naturally consists of a triple of manifolds $(B, \partial B, L)$ such that

(i) $B$ is contractible of $\operatorname{dim} m-2 n+3(m-4 n+4, m-8 n+6)$,

(ii) $\partial B$ is the image of singular orbits,

(iii) $L$ is the fixed point set which is a closed manifold of codimension $2(3,5)$ in $\partial B .(B, \partial B, L)$ is called the orbit triple of $\phi$.

THEOREM 5. The differentiable action $\phi$ of $\mathrm{SO}(n)(\mathrm{SU}(n), \mathrm{Sp}(n))$ on $a$ homotopy sphere $\Sigma^{m}$ for $n \geqq 11(n \geqq 8), 2 n-1<m<3 n-6(4 n-1<m$ $<6 n-9,8 n-1<m<12 n-15)$ with $V_{n, 2}\left(W_{n, 2}, X_{n, 2}\right)$ as the principal orbit is completely determined by the "orbit triple" $(B, \partial B, L)$ of $\phi$.

Combining the results of [5], [7], [9] with Theorems 4 and 5, we have theoretically completed the classification of differentiable actions of $\mathrm{SO}(n), \mathrm{SU}(n)$ and $\mathrm{Sp}(n)$ on homotopy spheres in the following range:

$$
\begin{aligned}
& \mathrm{SO}(n) \text { actions on } m \text {-spheres for } n \geqq 11 \text { and } m<3 n-6, \\
& \mathrm{SU}(n) \text { actions on } m \text {-spheres for } n \geqq 8 \text { and } m<6 n-9, \\
& \mathrm{Sp}(n) \text { actions on } m \text {-spheres for } n \geqq 8 \text { and } m<12 n-15 .
\end{aligned}
$$

\section{REFERENCES}

1. A. Borel et al., Seminar on transformation groups, Annals of Mathematics Studies, No. 46, Princeton Univ. Press, Princeton, N. J., 1961.

2. A. Borel, Fixed points of elementary commutative groups, Bull. Amer. Math. Soc. 65 (1959), 322-326.

3. A. Borel and F. Hirzebruch, Characteristics classes and homogeneous spaces. I, Amer. J. Math. 80 (1958), 485-538. 113.

4. G. Bredon, Examples of differentiable group actions, Topology 3 (1965), 103-

5. - Transformation groups on spheres with two types of orbits, Topology 3 (1965), 115-122.

6. W. C. Hsiang and W. Y. Hsiang, Some differentiable actions of $S^{1}$ and $S^{2}$ on 11-spheres, Quart. J. Math. Oxford Ser. (2) 15 (1964), 371-374.

7. - Classification of differentiable actions on $S^{n}, R^{n}$ and $D^{n}$ with $S^{k}$ as the principal orbit type, Ann. of Math. (to appear).

8. W. C. Hsiang, $A$ note on free differentiable actions of $S^{1}$ and $S^{3}$ on homotopy spheres, Ann. of Math. (to appear).

9. W. Y. Hsiang, On the classification of differentiable $\mathrm{SO}(n)$ actions on simply connected $\pi$-manifolds, Amer. J. Math. (to appear).

10. W. C. Hsiang and R. H. Szczarba, On the tangent bundle of a Grassman manifold, Amer. J. Math. 86 (1964), 685-697.

11. D. Montgomery and C. T. Yang, The existence of slice, Ann. of Math. 65 (1957), 108-116. 
12. G. D. Mostow, Equivariant embeddings in Euclidean space, Ann. of Math. 65 (1957), 432-446.

13. P. A. Smith, Fixed points of periodic transformations, Algebraic topology, Amer. Math. Soc. Colloq. Publ. Vol. 27, Amer. Math. Soc., Providence, R. I., 1942; Appendix B.

14. N. E. Steenrod, The topology of fibre bundle, Princeton Univ. Press, Princeton, N. J., 1951.

INSTITUTE FOR ADVANCED STUDy,

YALE UNIVERSITY AND

BROWN UNIVERSITY 